\title{
Modelos artesanales de simulación para el aprendizaje laparoscópico
}

\author{
Lopes Salazar A, Ramírez M*, Ruiz Cerdá JL.* \\ Servicio de Urología. Hospitales Luxemburgo y Mário Penna de Belo Horizonte. Brasil. \\ *Servicio de Urología. Hospital Universitario La Fe. Valencia.
}

Actas Urol Esp 2006; 30 (5): 457-460

\section{RESUMEN}

MODELOS ARTESANALES DE SIMULACIÓN PARA EL APRENDIZAJE LAPAROSCÓPICO

La laparoscopia representa un nuevo abordaje para la cirugía urológica ofreciendo una serie de ventajas. Su aprendizaje es un aspecto fundamental. Para la instauración en la práctica diaria es necesario mucho entrenamiento. El tiempo empleado en la curva de aprendizaje sigue siendo realmente largo y las maneras de entrenamiento escasas.

En la actualidad existen los modelos artesanales de simulación, que son aparatos sencillos y de bajo costo, además que pueden ser utilizados en cualquier sitio, facilitando el entrenamiento.

Hicimos una revisión en la literatura reciente acerca de estos modelos artesanales, que pueden en mucho facilitar el inicio del aprendizaje laparoscopio.

Palabras clave: Cajas artesanales. Aprendizaje laparoscópico.

\section{ABSTRACT}

TRADITIONAL TRAINING DEVICES FOR LAPAROSCOPY

Laparoscopy is a new approach to urological surgery with a number of advantages. The learning of this technique is very important. In order to establish this technique as routine practice a considerable amount of training is needed. The learning curve is still very long and training methods are scarce.

At the present time there are simple, low-cost simulators available that may be used in any place, thus facilitating training.

We have reviewed recent literature on these models, which can be of great help in initial laparoscopic training.

Keywords: Training boxes. Laparoscopic training.

$\mathrm{L}$ a laparoscopia representa en la actualidad un nuevo abordaje para la cirugía urológica que ofrece una serie de ventajas. Entre otras, una menor morbilidad, un efecto estético mejor y una vuelta a la vida normal más rápida. En la patología renal está muy bien establecida y puede ser considerada como estándar. En la prostática, es una opción alternativa a la cirugía abierta para determinados cirujanos expertos. En la cirugía vesical representa aún una alternativa lejana para la mayoría de urólogos.
$\mathrm{El}$ aprendizaje de la laparoscopia es un aspecto fundamental en su desarrollo e instauración en la práctica diaria. Hay que tener en cuenta que, para su realización son necesarias varias habilidades psicomotoras totalmente distintas de la cirugía abierta. Además, aunque se va acortando cada día más, el tiempo empleado en la curva de aprendizaje sigue siendo realmente largo. Sin embargo, estos factores no son los únicos que condicionan el aprendizaje laparoscópico. Ya que, antes de llegar a plantearse pasar a la 
cirugía in vivo, es necesario realizar una labor de entrenamiento previo fuera del ambiente quirúrgico. Para ello, son indispensables modelos o sistemas que reproduzcan artificialmente las condiciones de visualización, orientación espacial, coordinación y manipulación instrumental que el cirujano se va a encontrar posteriormente.

\section{MÉTODOS TRADICIONALES DE APRENDIZAJE}

Básicamente, existen tres modalidades de sistemas de simulación laparoscopia, los simuladores orgánicos, los simuladores de realidad virtual y las cajas de simulación ${ }^{1}$ (Tabla 1).

\section{Simuladores orgánicos}

Son considerados la mejor categoría una vez que reproducen exactamente las condiciones anatómicas, de irrigación sanguínea y de sensibilidad de los tejidos que van a ser manipulados. Pueden ser en humanos o animales, y en vivo o en cadáveres. En esta categoría tiene como desventajas la necesidad de un espacio especial para su realiza- ción, como un laboratorio propio, la poca accesibilidad y su elevado costo. Además, están también los aspectos éticos que conlleva la utilización de animales vivos para experimentación.

\section{Simuladores inorgánicos}

Se encuentran representados por dos categorías distintas: los simuladores de realidad virtual y los denominados simuladores sintéticos. Son sistemas muy útiles, una vez que pueden ser utilizados para entrenamiento en general y también para entrenamiento de habilidades especificas con ejercicios propios.

Los sistemas de realidad virtual aumentan cada día su valor en el entrenamiento urológico, varios centros de residencia norteamericanos utilizan esta herramienta como forma de aprendizaje. Estos sistemas son utilizados no sólo para el entrenamiento laparoscopio, pero también para entrenamiento de cirugias endoscopias en general. A pesar de representar una categoría de gran utilidad, aún constituyen una categoría con poca accesibilidad pues su precio es muy alto.

Tabla 1

Tipos de simuladores, ventajas y desventajas

\begin{tabular}{|c|c|c|}
\hline Tipo de simulación & Ventajas & Desventajas \\
\hline \multicolumn{3}{|l|}{ Orgánicas } \\
\hline \multicolumn{3}{|l|}{ Humanos } \\
\hline \multirow[t]{3}{*}{ En vivo } & \multirow{3}{*}{$\begin{array}{l}\text { Mejor categoría: reproduce exactamente la } \\
\text { anatomía del ambiente operatorio }\end{array}$} & Consideraciones éticas \\
\hline & & Necesita de consentimiento \\
\hline & & Presión del entrenamiento y la necesidad de tratamiento \\
\hline \multirow[t]{2}{*}{ Cadáver } & \multirow{2}{*}{$\begin{array}{l}\text { Gran fidelidad, misma anatomía, sin } \\
\text { problemas éticos, sin presión del tiempo }\end{array}$} & Alto costo \\
\hline & & $\begin{array}{l}\text { Alteraciones en la irrigación sanguínea y la } \\
\text { consistencia de los tejidos }\end{array}$ \\
\hline \multicolumn{3}{|l|}{ Animal } \\
\hline \multirow[t]{3}{*}{ En vivo } & \multirow[t]{3}{*}{ Gran fidelidad de tejidos y hemorragia } & Problemas éticos \\
\hline & & Alteraciones anatómicas \\
\hline & & Alto costo \\
\hline \multirow[t]{4}{*}{ Cadáver } & \multirow{4}{*}{ Costo bajo, gran fidelidad de los tejidos } & \\
\hline & & Alteraciones anatómicas y sin hemorragia \\
\hline & & Alto costo \\
\hline & & Potencial de contaminación \\
\hline \multicolumn{3}{|l|}{ Inorgánicos } \\
\hline \multirow[t]{2}{*}{ Realidad Virtual } & \multirow{2}{*}{$\begin{array}{l}\text { Capacidad de realizar ejercicios en tiempo } \\
\text { real, feedback instantáneo y objetivo }\end{array}$} & Equipamientos muy caros \\
\hline & & $\begin{array}{l}\text { Eficacia de la transferencia de fidelidad del software y } \\
\text { el operador cuestionable }\end{array}$ \\
\hline Sintéticos & $\begin{array}{l}\text { Gran fidelidad. Puede ser utilizado para } \\
\text { entrenamiento o ejercicios específicos }\end{array}$ & Modelos de alta fidelidad son de alto costo \\
\hline
\end{tabular}


En la categoría de los simuladores sintéticos existen dos tipos: los profesionales, que representan aparatos de alta fidelidad y se encuentran comercializados, y una nueva modalidad, los aparatos artesanales o custom made con la posibilidad de ser construidos por los propios urólogo. Las primeras son sistemas muy bien establecidos en el mercado y de gran calidad. Sin embargo, el gran problema es su elevado costo económico. En muchas ocasiones alcanzan los 1.000 euros, por lo que estos sistemas quedan reservados a instituciones. Además, limitan el tiempo, la disponibilidad y el fácil acceso al uso por el urólogo. Los simuladores comerciales más conocidas en el mercado son: el vídeo pelvitrainer y los simuladores de dos espejos, el Simuview.

Los simuladores o sistemas artesanales de aprendizaje laparoscopio, representan una categoría relativamente nueva, puesto que son modelos compuestos de componentes fáciles de adquirir con los que se pueden lograr construir aparatos de entrenamiento muy útiles y confiables. Los sistemas más populares son compuestos por una cámara de vídeo sencilla, como una webcam, conectada a un ordenador portátil, una fuente de luz común, como una lámpara incandescente y una caja para simular el ambiente cerrado de trabajo. Utilizando instrumentos laparoscopias ya usados y limpiados, se puede entonces practicar los pasos y movimientos que reproduzcan la técnica operatoria ${ }^{2}$.

Los modelos artesanales de simulación laparoscopia son conocidos desde hace más de 10 años. En la actualidad, con la facilidad de los ordenadores portátiles y las cámaras (webcans) de bajo costo, surgen modelos más accesibles y de mejor calidad ${ }^{3}$. Algunos de ellos utilizan aparatos de alta tecnología, por ejemplo con cámaras que poseen la capacidad de foco automatizado, con aproximación y el sistema de face-tracking, que acompaña los movimientos del cirujano haciendo el papel de un ayudante. De esta forma, se ha aumentado aún más la calidad del trabajo de estos aparatos ${ }^{2}$. Beatty, en el 2005, describió un modelo artesanal, que además de utilizar una webcam, también utiliza una caja translúcida que no necesita de iluminación para su utilización. Lo que hace más sencillo aún el aparato de entrenamiento ${ }^{2}$ (Fig. 1).
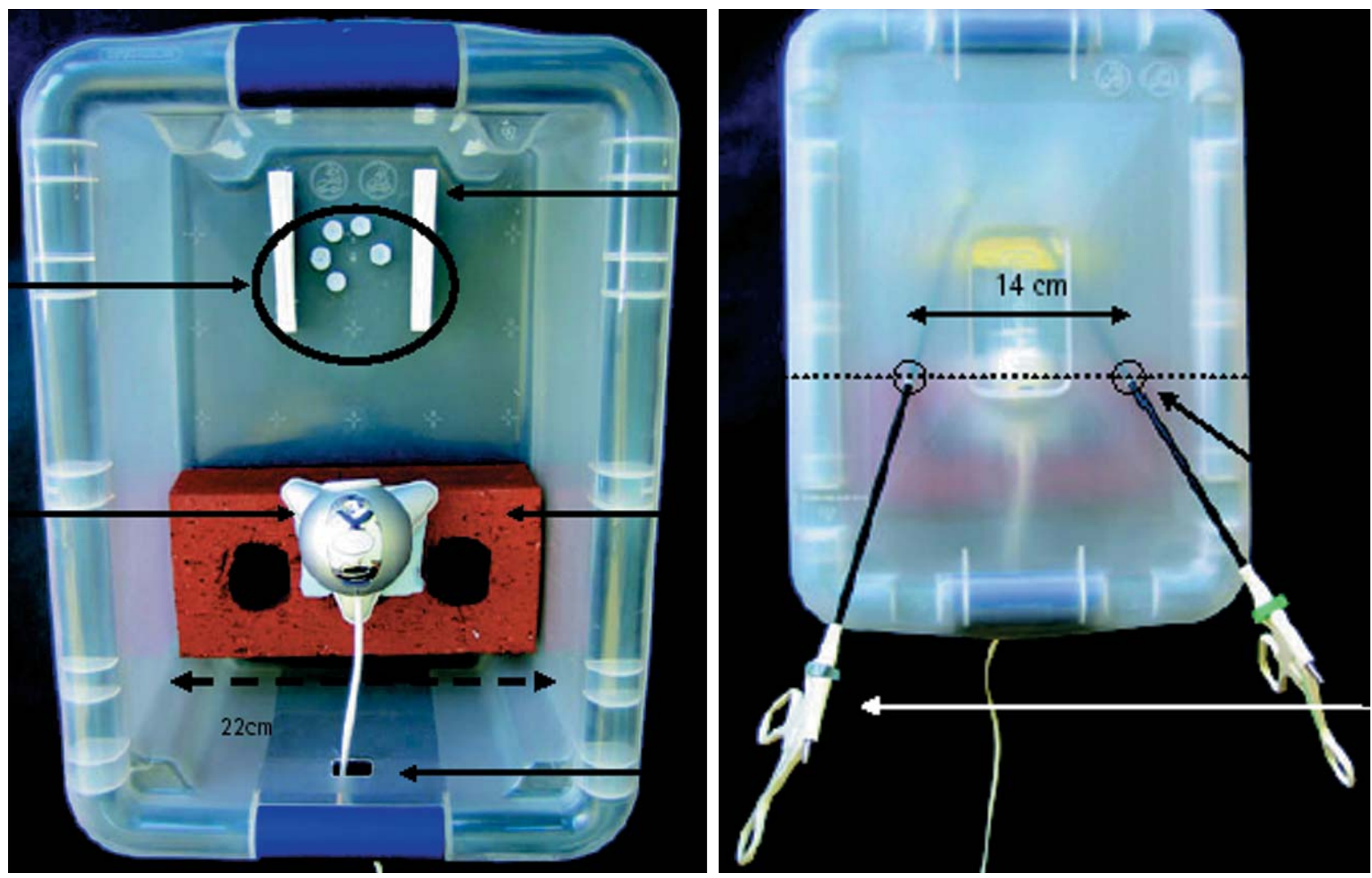

FIGURA 1. Modelo de entrenamiento laparoscópico que utiliza una webcam y una caja translúcida. Beaty, BJU - 2005. 
Los modelos artesanales de simulación además de sencillos y de bajo costo, poseen otras ventajas. Ya que, pueden ser utilizados en cualquier sitio, de esta forma el entrenamiento puede ser realizado fuera del ambiente del hospital o en casa, aumentando aún más el tiempo de entrenamiento. Existen varios ejercicios que pueden ser realizados para entrenamiento de habilidades específicas. Por otra parte, cuando se usa una webcam conectada a un ordenador es posible grabar los ejercicios realizados para una revisión posterior. También quedan a disposición de otros cirujanos para ser compartidos a través de intranets o Internet ${ }^{2}$.

\section{CRITERIOS PARA VALORAR LOS SIMULADORES}

El gran problema de los simuladores para el entrenamiento laparoscópico es la dificultad para evaluarlos. No existe un consenso acerca de cual es el método más efectivo. Sólo se sabe que dos criterios esenciales para que un aprendiz tenga acceso a este entrenamiento con facilidad son que el sistema sea de bajo costo y posea gran viabilidad. Chung et al. hicieron un estudio en el que compararon la efectividad de un simulador tipo box trainer con los modelos de realidad virtual. Los participantes no tenían práctica laparoscopia previa. Se estipularon ejercicios para medir el tiempo en realizar los ejercicios y la mejoría personal de cada cirujano. Llegaron a la conclusión de que no había diferencia entre los dos métodos y que también no había una manera eficaz de valoración de la mejoría de la técnica, ni para establecer el tiempo mínimo de práctica para llegar a un resultado satisfactorio ${ }^{3}$.

No existe aún consenso acerca de las herramientas de evaluación del entrenamiento laparoscopio. Feldman et al., en el 2004, hicieron una revisión de la literatura acerca de las maneras de evaluar el entrenamiento laparoscopio con simuladores en cuanto a su competencia ${ }^{4}$. La habili- dad quirúrgica es una resultante de una mezcla de conocimiento teórico, juicio y habilidad técnica. Todos estos conceptos se ponen muy de relieve en el comienzo del entrenamiento laparoscopio. Sin embargo, hace que sea difícil su evaluación. Estos autores llegaron a la conclusión de que los aparatos de simulación son útiles para el entrenamiento de cirujanos no experimentados y para el entrenamiento de las habilidades básicas. Sin embargo, no son completos para toda la formación de un cirujano laparoscopio.

\section{CONCLUSIÓN}

En la actualidad, la cirugía laparoscopia representa una modalidad muy utilizada que proporciona grandes benéficos para los pacientes. Su aprendizaje es especial, teniendo en cuenta que son necesarias habilidades distintas de la cirugía abierta. Existen varias modalidades de simuladores para el entrenamiento de la cirugía laparoscopia, los que están más populares son los simuladores artesanales, que son de bajo costo y pueden ser utilizados en casa. A pesar del uso de estos simuladores no hay nada comprobado de cual es la mejor categoría, pero para el entrenamiento del cirujano no experimentado son muy eficaces.

\section{REFERENCIAS}

1. Munz Y, Kumar K, Moorthy S, Bann S, Darzi A. Laparoscopic virtual reality and box trainers: is one superior to the other?. Surg Endosc. 2004; 18:485-494.

2. Beatty JD. How to build an inexpensive laparoscopic webcam-based trainer. BJU International. 2005;96:679-682.

3. Chung YS, Landsittel D, Chon HC, NG CS, Fuchs JG. Laparoscopic skills training using a webcam trainer. J Urol. 2005; 173:180-183.

4. Feldman LS, Sherman V, Fried GM. Using simulators to assess laparoscopic competence: Ready for widespread use?. Surgery. 2004;135:28-42.

Dr. A. Lopes Salazar

E-mail: salazar2175@hotmail.com 